\title{
Povidone-iodine chemical pleurodesis in treating spontaneous chylothorax in pediatric patients
}

\author{
Wei-Guang Long, Bin Cai, Yang Liu, Wen-Jie Wang \\ Department of Chest Wall Surgery, Guangdong Second Provincial General Hospital, Guangzhou 510317, China \\ Contributions: (I) Conception and design: WG Long; (II) Administrative support: WG Long; (III) Provision of study materials or patients: None; (IV) \\ Collection and assembly of data: B Cai, Y Liu, WJ Wang; (V) Data analysis and interpretation: WG Long; (VI) Manuscript writing: All authors; (VII) \\ Final approval of manuscript: All authors. \\ Correspondence to: Wei-Guang Long. Department of Chest Wall Surgery, Guangdong Second Provincial General Hospital, Guangzhou 510317, \\ China. Email: xlg800@hotmail.com.
}

Background: Chylothorax is a rare disease with a high mortality rate in pediatric patients like newborns and infants. As one of the minimally invasive treatments, chemical pleural pleurodesis has been gradually used in treating pediatric chylothorax patients in recent years. This study explored the feasibility, safety and effect of povidone-iodine chemical pleurodesis (CP) for spontaneous chylothorax in young pediatric patients. Methods: From January 2009 to December 2019 the clinical data of 22 children treated for spontaneous chylothorax at Guangdong Second Provincial General Hospital were retrospectively analyzed. The data included the patients' medical history, clinical manifestations, pleural effusion examinations, treatment methods, and effects and complications. On the basis of conventional conservative treatment and closed thoracic drainage, all the children were treated by CP with povidone-iodine until the pleural effusion was absorbed and the thoracic tube was removed. After discharge from the hospital, each patient was followed up for 1-10 years.

Results: Of the 22 children (16 males and 6 females), 3 cases were diagnosed with pleural effusion at prenatal examination, 5 cases had acute respiratory distress at birth, and 6 case had experienced pneumonia repeatedly since birth. All of the children had different degrees of cyanosis and dyspnea. Pleural effusion was diagnosed in all patients by chest X-ray and chest ultrasound after admission (12 cases on the left side, 5 cases on the right side, and 5 cases on both sides). The results of all chyle tests during pleural effusion examination were positive. The mean leukocyte count was $(9,278.8 \pm 9,504.6) \times 10^{6} / \mathrm{L}$, the lymphocyte ratio was $(83.9 \pm 6.1) \%$, and the mean triglyceride content was $(7.18 \pm 6.10) \mathrm{mmol} / \mathrm{L}$. All patients were treated with thoracic drainage, diet control, nutritional support, and povidone-iodine CP, among 6 of them with pneumonia on admission received antibiotics. The mean length of stay (LOS) was $(36.0 \pm 18.2)$ days. The patients received thoracic drainage for $(23.0 \pm 15.6)$ days and pleurodesis $(3.0 \pm 2.0)$ times. No serious side effects occurred after pleurodesis, although some patients experienced mild chest pain and low fever. All the patients successfully achieved a clinical cure.

Conclusions: Povidone-iodine CP is a safe, effective, and minimally invasive treatment, with high success rate and few complications in young pediatric patients with spontaneous chylothorax.

Keywords: Chylothorax; pleural effusion; pleurodesis; povidone-iodine

Submitted Mar 08, 2020. Accepted for publication Apr 27, 2020.

doi: 10.21037/apm-20-926

View this article at: http://dx.doi.org/10.21037/apm-20-926 


\section{Introduction}

Chylothorax is a rare disease that occurs when chylous lymphatic fluid accumulates in the thoracic cavity. Congenital chylothorax, is one of the most common causes of neonatal pleural effusion in young children, with an incidence of 1 in 6,000 to 24,000 live births. The condition carries a high mortality rate up to $60 \%$ and affects boys more than girls, at a ratio of about 2:1, Premature delivery, fetal edema, low birth weight, hypoplasia of lung, hypoproteinemia, or persistent chylous exudation are associated with congenital chylothorax and can all increase the risk of death (1-4). For 5\% to $10 \%$ of chylothorax patients, the cause is not identified; this is known as idiopathic chylothorax. In this study, both types of chylothorax described above were classified as spontaneous chylothorax by the author.

Chylothorax has been clinically reported for almost a century. At present, it is mainly treated through conservative and minimally invasive approaches such as basic life support, thoracic puncture, closed thoracic drainage, and chemical pleural pleurodesis, etc., and few patients undergo surgical treatment such as ligation of thoracic duct (5). As a consequence of the low incidence rate and lack of relevant research into spontaneous chylothorax, the etiology of most spontaneous chylothorax is still unknown, and no clear clinical pathway, expert consensus, or guidelines exist in China or elsewhere.

In recent years, povidone-iodine chemical pleurodesis (PICP) is expected to be a new minimally invasive treatment for pediatric spontaneous chylothorax following the reports of some successful cases. Povidone-iodine (PI) has been used for chemical pleurodesis (CP) treatment of pneumothorax or refractory pleural effusion for a long time. Its safety has been confirmed in adult patients, so it is expected to be used in pediatric patients. Based on the previous researches, we tried to improve the PICP treatment by regulating the volume and concentration of PI sclerosant, aiming to evaluate the safety and efficacy of this technique and explore a more effective conservative treatment. From January 2009 to December 2019, a retrospective analysis was carried out of the cases of spontaneous chylothorax in young patients treated by PICP in chest wall surgery at our hospital, and the report is as follows. We present the following article in accordance with the STROBE reporting checklist (available at http://dx.doi.org/10.21037/apm-20-926).

\section{Methods}

\section{Patients}

Between January 2009 and December 2019, young chylothorax patients treated at our hospital were enrolled in the study. Patients who met the following criterias were included: (I) aged $\leq 7$ years old; and (II) pleural effusion was confirmed by chest X-ray, chest ultrasound, and other examinations. Patients who met any of the following criteria were excluded: (I) a history of asphyxia, trauma, or surgery; (II) other congenital diseases and genetic diseases, or diseases clearly related to chylothorax; or (III) intrathoracic operations other than thoracentesis or closed thoracic drainage were performed.

After screening, 22 patients met the criteria, including 18 cases who were transferred to our department after closed thoracic drainage at other hospitals. Their data, including their age, gender, basic disease information, examination results of hydrothorax, treatment, curative effect, and any complications, were retrospectively analyzed.

Prior to the treatment, written informed consent was obtained from each patient's legal guardian. This study was conducted in compliance with the Declaration of Helsinki and received approval from the ethical committee of Guangdong Second Provincial General Hospital.

\section{Diagnosis of chylothorax}

All of the 22 patients were diagnosed with chylothorax based on the following: (I) chyloid pleural effusion was drained by thoracentesis or closed thoracic drainage; (II) chyloid test was positive in pleural effusion during the normal lactation period (formula milk or breast milk); (III) pleural effusion examination: leukocyte count $1,000 / \mathrm{mL}$ $\left(1.0 \times 10^{6} / \mathrm{L}\right)$, lymphocyte percentage $\geq 70 \%$, triglyceride $\geq 1.24 \mathrm{mmol} / \mathrm{L}$, and bacterial culture was negative $(6,7)$.

\section{Basic support treatment}

After admission, all of the eligible children underwent electrocardiographic monitoring, oxygen inhalation, and closed thoracic drainage. Cytological examination, biochemical analyses, bacteria culture, and chyle testing were performed on the pleural fluids, while routine blood, 
urine and feces, biochemical, coagulation function, blood type, thyroid function, hepatitis B virus, and C-reactive protein (CRP) tests were performed. The patients also underwent ECG, chest X-ray, and chest ultrasound.

After contraindications were excluded, a doublelumen central venous catheter (CVC) was placed under sevoflurane anesthesia. A small amount of enteral nutrition was maintained during the treatment process: $1 / 4$ to $1 / 3$ of the daily demand of milkfat products (such as formula milk powder) and $1 / 3$ to $1 / 2$ of the daily demand of liquid were given. Then, a parental nutrition solution based on the age and weight of the child, comprising glucose, fat emulsion, amino acid, and vitamins according to the remaining daily nutritional demand, was pumped into the CVC to complete the nutrition support. Electrolytes, human albumin, and immunoglobulin were given according to the results of blood tests. Cefazolin sodium and cefuroxime axetil granules were administered to treat pneumonia. Blood tests, blood biochemical tests, CRP, chest X-ray, and chest ultrasound were performed routinely.

\section{Povidone-iodine CP}

The patients' pleural effusion condition was monitored daily until it became lighter and the daily amount gradually decreased. $\mathrm{CP}$ was feasible at this time. The patients were reexamined by chest $\mathrm{X}$-ray and ultrasonography to confirm the smooth drainage of the pleural effusion, and that the affected side of lung was completely re-expanded.

For PICP, PI sclerosant was produced by mixing $25 \mathrm{~mL}$ of $0.1 \%$ PI and $25 \mathrm{~mL}$ of normal saline together at a ratio of 1:1. Then, a small amount of normal saline was used to clean the chest tube and drain the remaining pleural effusion to ensure that the chest tube could drain with without obstruction. The vital signs of the patients were monitored by electrocardiogram monitor and observed to be stable. The sclerosant was then injected slowly through the chest tube when the children were calm. During the injection process, the children were closely monitored for their vital signs and symptoms related to dyspnea and pleural reaction. Depending on the reaction of the child's response, the sclerosant was then partially or completely injected. After the injection, the chest tube was connected to the drainage bag for continuous drainage, and the patients' guardians were asked to help change their position, so that the sclerosant could reach all parts of the pleura.

After the procedure, the patients' conditions were observed closely, and their chest X-rays or ultrasounds were rechecked within 24 hours. If pleural effusion had recurred, the basic support treatment was continued and PICP was performed again. If the pleural effusion was significantly reduced or had disappeared, the drainage tube was clamped after the effusion was completely drained, the basic support treatment was continued, and the patients' chest X-rays or chest ultrasounds were regularly checked. If the amount of pleural effusion was increased after reexamination, the chest tube was opened to continue drainage and continue treatment according to the recurrence of pleural effusion. If the following clinical cure criterias were met, the patients were considered to be clinically cured. After discharge, the cured children were kept on a low-fat diet. One and three months after discharge, the children's chest X-rays or chest ultrasounds were checked. If the pleural effusion had not recurred, the child could gradually return to their normal diet. The patients were followed up for 1-10 years. See Table 1 for treatment details.

\section{Clinical cure standards}

After treatment with PICP, the pleural effusion was basically absorbed with no increases for 1 to 2 weeks, and there were no serious adverse reactions in any of the children; the blood examination indexes were basically restored to normal, and the thyroid function was normal.

\section{Statistical analysis}

Data analyses were performed using IBM SPSS Statistics software (Version 22.0. IBM Corporation, NY, USA), and the mean \pm standard deviation was used for measurement data.

\section{Results}

\section{Patient clinical characteristics}

After screening, a total of 22 children, including 16 males and 6 females, with spontaneous chylothorax met the criteria. The patients were aged between 21-day-old and 87-month-old.

Pleural effusion was confirmed by chest radiograph and chest ultrasound, and included 12 and 5 cases on the left and right sides, respectively, and 5 cases on both sides. Pleural effusion was found during prenatal ultrasound examination in 3 cases, and 5 of the patients were born with acute respiratory distress. After high flow oxygen and hormone 
Table 1 Clinical and therapeutic characteristics and results of pleural effusion examination of pediatric patients with spontaneous chylothorax

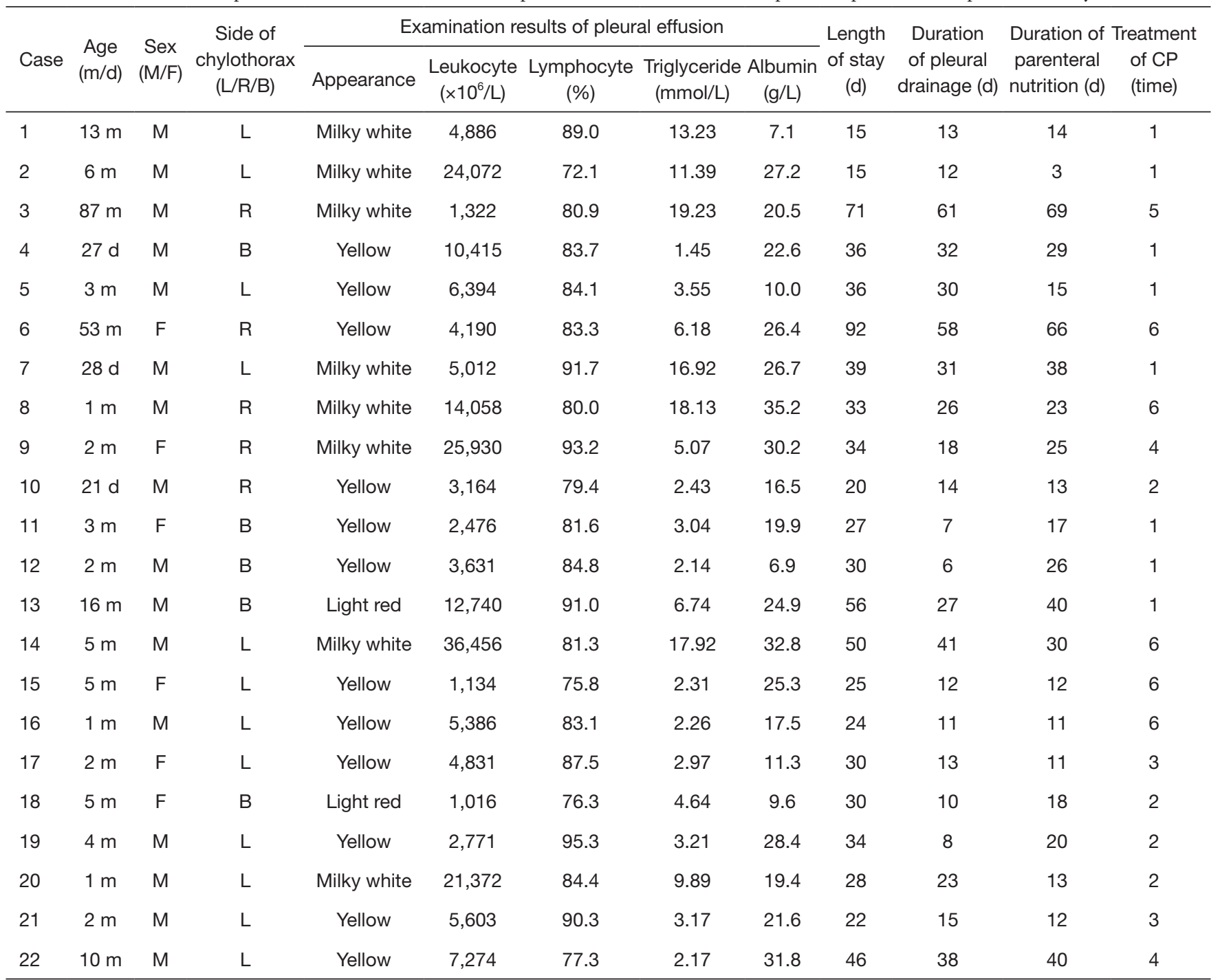

m/d, months/days; M/F, male/female; L/R/B, left/right/bilateral; CP, chemical pleurodesis.

treatment, respiratory function recovered smoothly (without endotracheal intubation or the assistance of a ventilator). Six cases had suffered pneumonia repeatedly since birth.

After examining the pleural effusion, all the children were confirmed as chylothorax. The chylothorax tests from the children were positive, the leukocyte count was $(9,278.8 \pm 9,504.6) \times 10^{6} / \mathrm{L}$, the lymphocyte ratio was $(83.9 \pm 6.1) \%$, and the triglyceride content was $(7.18 \pm 6.10) \mathrm{mmol} / \mathrm{L}$. See Table 1 for examination results.

\section{After basic support treatment}

During the basic support treatment, all of the children had stable vital signs and body weight. None of the children experienced complications related to anesthesia, bleeding, incisions, or catheter-related infection after indwelling closed thoracic drainage tubes and CVCs. The symptoms of anhelation and dyspnea were alleviated after the effusion was drained by closed thoracic drainage.

During the treatment, 7 cases of mild anemia and 5 cases of leukopenia were found by routine blood examination. After clinical recovery was achieved, the patient's blood examination indexes basically reached the normal range, and the thyroid function was normal. After diet control, the amount of pleural effusion gradually decreased to about one-half of the daily amount measured at the time 

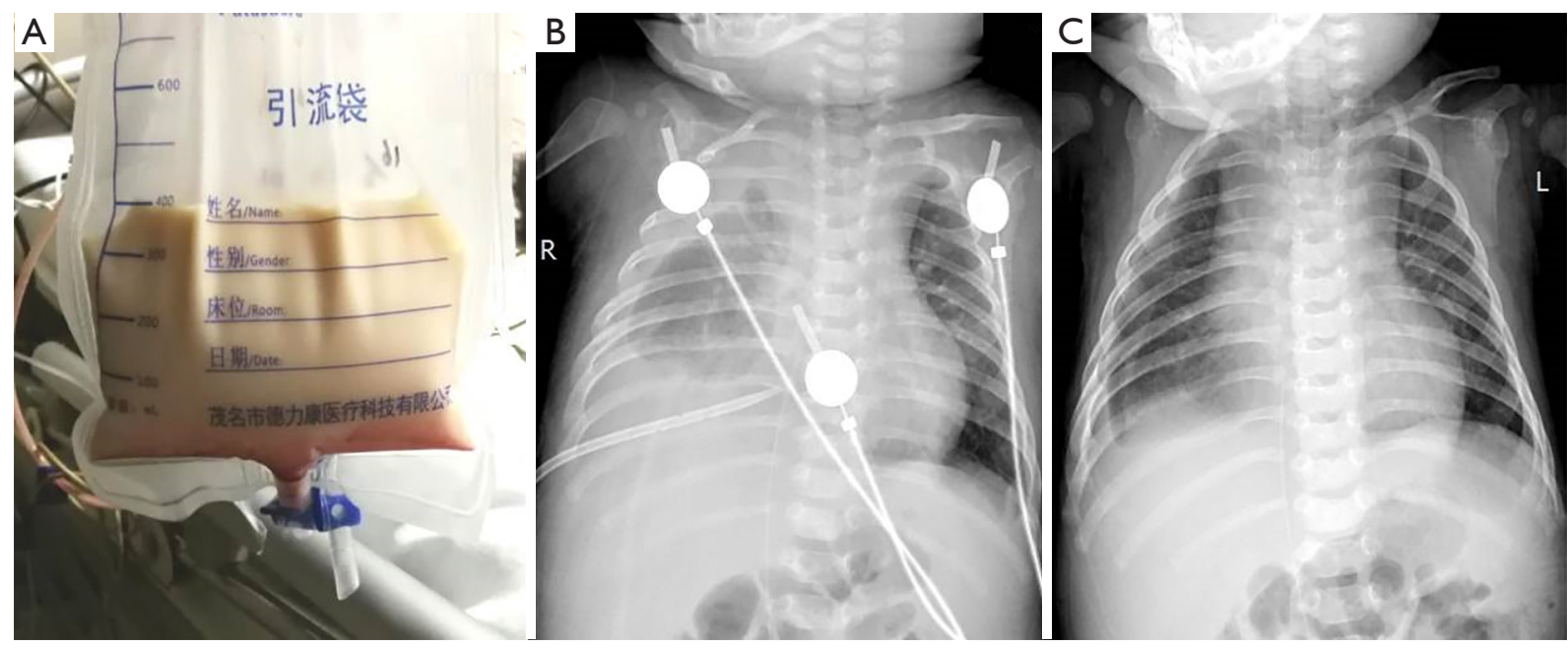

Figure 1 The pleural effusion and chest X-ray of case No. 8 before and after treatment. The chest X-ray examination result of case No. 8 showed a medium amount of pleural effusion in the right thoracic cavity (B), which was milky white in color (A). After treatment, the pleural effusion was basically absorbed $(\mathrm{C})$.

of admission, although it continued to exist and increased in line with oral intake. At the time of admission, 6 of the children were diagnosed with pneumonia, which was characterized by fever and expectoration, and was cured by atomization, expectorant, and anti-infection.

\section{After pleurodesis with povidone-iodine chemical}

All of the patients were treated with PICP. The dosage of sclerosant varied from 25 to $50 \mathrm{~mL}$, depending on the patient's condition. After the injection of sclerosant into the thoracic cavity, all of the children displayed reactions associated with chest pain, such as crying and restlessness, which occurred within seconds to minutes of the sclerosant injection and lasted between 1 hour and 1 day. Fever of varying degrees, lasting for 1 day to 3 days, was recorded in all of the children, with a maximum temperature of $38.8^{\circ} \mathrm{C}$. The children's temperatures returned to normal after physical cooling and antipyretic drugs, such as ibuprofen drops. A transient decrease of blood oxygen saturation was observed in 14 children, with a minimum drop to $93 \%$, with mild anhelation; this returned to normal and relieved after the sclerosant was drained out with the chest tube. During the treatment, all of the children's mental and physical activities were normal, eating and defecation were basically normal, and there were no other obvious uncomfortable symptoms observed. Chest X-ray and ultrasonography showed that the pleural effusion was absorbed in 22 children. Chest tubes in 17 cases $(77.3 \%)$ were removed within 1 month.

All of the patients were followed for 1 to 10 years after discharge. During the follow-up period, 1 patient (case 13) still exhibited a small volume of pleural effusion in the left pleural cavity during $\mathrm{X}$-ray evaluation; however, this was not increased compared with the chest $\mathrm{X}$-ray before discharge. The follow-up chest $\mathrm{X}$-rays of the other children showed that the pleural effusion had been completely absorbed. Each of the children resumed a normal diet with a satisfactory standard of daily living, without any symptoms of cardiac, pulmonary, or renal dysfunction, or dysthyroidism. Figures 1 and 2 show one case of pleural effusion and chest $\mathrm{X}$-ray after admission and clinical cure, respectively.

\section{Discussion}

$\mathrm{CP}$ is a therapeutic procedure which, via a chemical or biological sclerosant injection into the thoracic cavity, can induce aseptic pleurisy, and can create the adhesions between the parietal and visceral pleura and obliterate the thoracic cavity. At present, CP is mainly used as a minimally invasive treatment for patients with pneumothorax and pleural effusion (8), but its use is still rarely reported in the treatment of chylothorax.

In previous studies on CP, talc, OK-432, erythromycin, hypertonic glucose, and PI have been the main sclerosants 

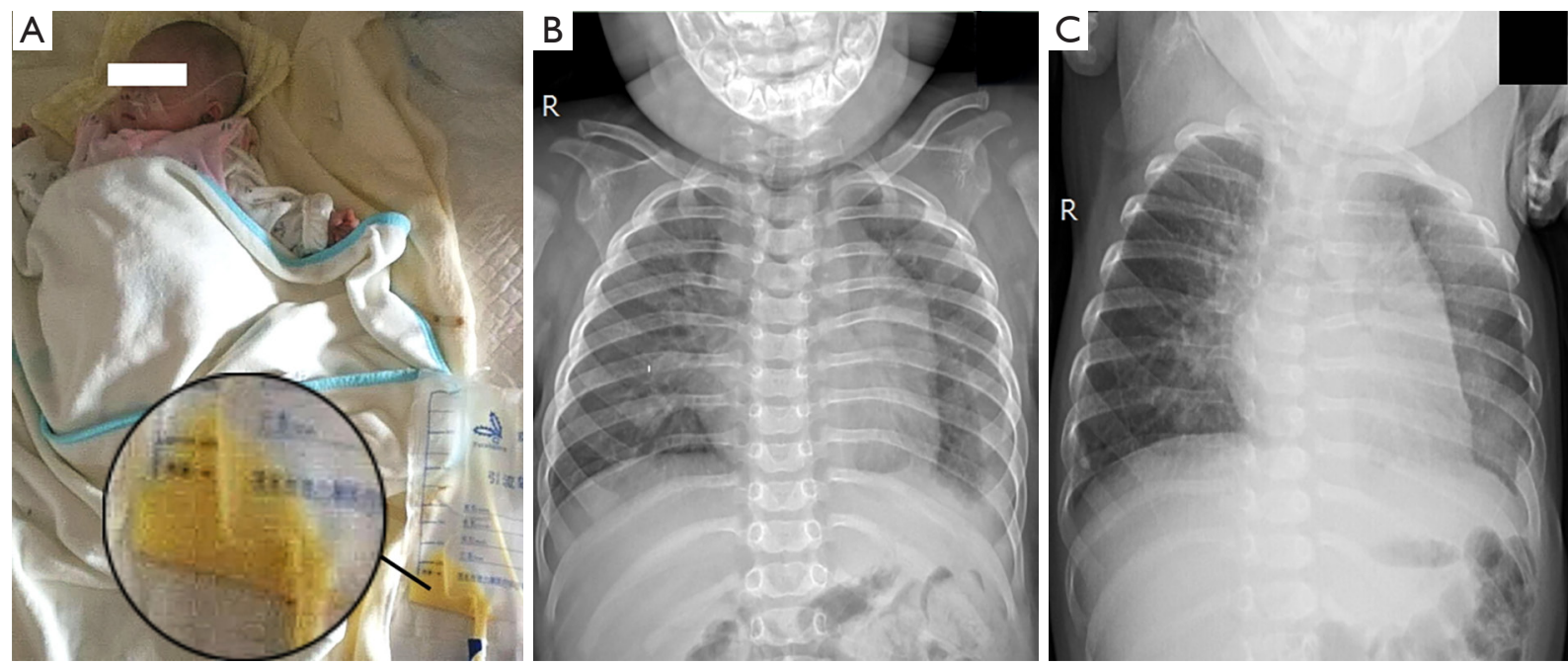

Figure 2 The pleural effusion and chest X-ray of case No. 7 before and after treatment. The pleural effusion of case No. 7 was milky white in color (A) and a small amount of pleural effusion in the left thoracic cavity at admission can be seen (B). The pleural effusion was completely absorbed after clinical cure $(\mathrm{C})$.

used $(9,10)$. Brissaud first reported using PICP to treat congenital chylothorax in 2003 (11). However, the efficacy of PI as a sclerosant has not been fully demonstrated up to now, as the incidence of chylothorax is low and there have been few related studies. Inspired by the above research, this study discussed the results of the improvement in the treatment of PICP, with the aim of describing clinical experience of the conservative and minimal invasive treatment of spontaneous chylothorax in children.

Spontaneous chylothorax differs from acquired chylothorax, which can be attributed to a definite cause such as surgical trauma, injury, or acquired disease. The etiology of spontaneous chylothorax is often unknown, but it is generally considered to be related to the abnormal development of the lymphatic system. Only $10-20 \%$ of spontaneous chylothorax is accompanied by lymphangiodilation, lymphangiomatosis, trisomy 21 syndrome, Noonan syndrome, and other diseases (12). At present, because its unknown causes and small number of cases, chylothorax still lacks definitive treatment protocols, and its treatment is divided into conservation and surgical treatment based on clinical experience.

Infants with chylothorax often have poor cardiopulmonary function, and usually require life support, thoracentesis, and closed thoracic drainage after birth. During the treatment process, drainage of chylothorax leads to the loss of lipids, proteins, fat-soluble vitamins and electrolytes as well as lymphocytes, antibodies and coagulation factors, resulting in malnutrition, dehydration and electrolyte disorder. This may also significantly increase the risk of nosocomial infection and septicemia (13). Therefore, life support is a basic element conservative treatment for chylothorax. The chest wall lymphatics of infants are small and have many collateral vessels. Treatment with lymphangiography, embolization, or ligation of thoracic duct is challenging in infants, and the risk of anesthesia is high. Moreover, previous reports have found that the success rate of conservative treatment for chylothorax is around $75 \%$ (5), especially in children with small chylous exudation; therefore, conservative treatment is the first choice at present. Thus, it is highly important to explore a safe and effective treatment to promote the absorption of pleural effusion and the repair of thoracic lymphatics.

Compared with surgery, $\mathrm{CP}$ is minimally invasive and straightforward to apply in the treatment of spontaneous chylothorax. First, most young pediatric patients with chylothorax require closed thoracic drainage to relieve the compression on the heart and lungs, hypoxia, and pulmonary inflammation caused by pleural effusion, meaning the chest tube has a very high utilization rate, and $\mathrm{CP}$ can be completed through the chest tube at the same time. Second, closed thoracic drainage is a minimally invasive operation with little trauma and wholly fits with the concept of enhanced recovery after surgery. In our study, a small diameter chest tube (12-16 $\mathrm{F}$, depending on the age of the children) was used. The patients experienced no obvious pain from the skin incision, and the pleural effusion was drained smoothly. 
During CP treatment, the sclerosant injected into the thoracic cavity causes a strong inflammatory reaction in the pleural mesothelium due to its physical and chemical properties, resulting the secretion of a large number of inflammatory mediators and chemotaxis of inflammatory cells (e.g., neutrophils and monocytes). Pleura inflammation leads to pleurisy hyperplasia, fibrosis, and finally thoracic cavity obliteration (8); this process is seen when CP is used to treat chylothorax, and is also used to treat pneumothorax and pleural effusion. Pleura inflammation can also affect the structure and function of lymphatic vessels in the chest wall.

Spontaneous chylothorax is often related to the abnormal development of lymphatics. When there is absence, atresia, or stenosis of lymphatics, its downstream branches are more likely to rupture under high pressure, with chylothorax as the result. However, the pressure stimulation also opens many communicating branches and promotes the formation of new collateral branches, which become alternative pathways for abnormal lymphatics. Therefore, closing the thoracic cavity and lymphatic laceration is not the only key to the successful treatment of spontaneous chylothorax: promoting the formation and growth of lymphatic collateral circulation also carries importance. Surgical ligation of the thoracic duct does not help to preserve the only drainage function of abnormal lymphatics, but may in fact inflict new damage to the chest wall lymphatics and, inevitably, surgical injury to the body during the operation. Moreover, there is great potential growth and development in young children. After CP treatment, a large number of inflammatory mediators produced in the process of pleural inflammatory reaction rapidly induce the proliferation of new lymphatics and capillaries $(14,15)$, which is conducive to the recanalization of the lymphatics in the chest wall and the promotion of the reabsorption and circulation of chyle.

PI, which is mainly used to disinfect the skin and mucosa in clinic, plays the role of a pleura obliterator, inducing pleurisy through strong oxidation and acidity ( $\mathrm{pH}=2-4)$. As early as 2012, Agarwal et al.'s (16) metaanalysis demonstrated the effectiveness of using PI for CP treatment, indicating that the success rates for treating recurrent pneumothorax and pleural effusion were $93.5 \%$ and $88.5 \%$, respectively. Moreover, it demonstrated a similar level of efficacy to talc, while, excluding chest pain, resulting in fewer adverse reactions. Matus et al. (17) reported that the success rate of PICP in treating malignant pleural effusion ranged from $79 \%$ to $82.2 \%$. In these studies, it was highlighted that the incidence of chest pain after CP was as high as $55 \%$ and $31 \%$ respectively, and attention should be paid to possible allergic reactions, cardiopulmonary insufficiency, thyrotoxicosis, nephrotoxicity, and visual impairment. However, other studies have shown that the above adverse reactions are usually rare, as long as the dosage, concentration, and time of PI are well controlled to avoid excessive absorption by the pleura during the treatment process (18).

Based on its high success rate, PICP has gradually been used to treat chylothorax. Borcyk et al. (19) and Hmami et al. (20) respectively reported that a child with repeated chylothorax who responded poorly to conservative therapies was cured by using $6 \mathrm{~mL}$ of $4 \%$ PI in the thoracic cavity for $5 \mathrm{~h}$ and $5 \mathrm{~mL}$ of $4 \%$ PI. Murki et al. (21) reported that using $4 \mathrm{~mL}$ of $4 \%$ PI diluted with $6 \mathrm{~mL}$ of normal saline into $10 \mathrm{~mL}$ and keeping in the thoracic cavity for 5 h by bilateral CP 3 times cured a child with congenital chylothorax. None of the above three studies reported any PI-related adverse reactions. However, based on previous findings regarding the adverse reactions of PI, they suggested monitoring thyroid and renal function. In Resch's report (22), the treatment success and survival rates of 12 children with congenital chylothorax treated with PICP using $4-5 \mathrm{~mL}$ of $4-10 \%$ PI for $1-5 \mathrm{~h}$ were $58 \%$ and $75 \%$, respectively. Two of cases who died were diagnosed with diffuse lymphangiectasis (DLA), which has been reported to potentially lead to excessive absorption of PI in the pleura during CP treatment and a subsequent significant increase in the incidence of related adverse reactions, eventually resulting in death (19).

Based on the conclusions of previous research, this study improved the treatment of chylothorax by reducing the concentration of PI, increasing the total amount of sclerosant, and shortening the retention time. Reducing the dosage of PI can reduce the probability of adverse reactions. Moreover, depending on the patient's tolerance, properly increasing the total amount and shortening the retention time of sclerosant can help it to fully reach all parts of the pleura, and quickly create adhesions between the parietal and visceral pleura after pleurisy. Through this, adhesion is evenly formed and the probability of local encapsulated pleural effusion is reduced, which is fits with the principle of CP treatment that creating an extensive pleural adhesion and obliterating the thoracic cavity completely $(8,23)$. Furthermore, PI works extremely quickly, killing most of the cells it comes into contact with within $30 \mathrm{~s}$ in vitro. Therefore, after PI sclerosant is injected into the pleural cavity, it can be drained slowly without the chest tube being clamped, which not only shortens the time for the pleura 
to absorb the PI and reduces the probability of adverse reactions, but also reduces the concurrent chest pain to some degree. In addition, PI absorbed by the pleura can be excreted through urine in short time after PICP. Unlike some sclerosants (e.g., antibiotics), PI does not need to be metabolized through liver that can protect the liver function of children. At last, PI is inexpensive and easy to obtain in hospitals.

In this study, 22 cases were clinically cured by PICP. To protect the intestinal mucosal barrier and the digestion function, children were allowed a small amount of low-fat food without fasting. As a result, a small amount of pleural effusion always recurrence, and chylothorax could not be completely cured by basic support treatment because most of the chyle came from the lymphatic in small intestine. After PICP, complete absorption of the pleural effusion in all cases could prove that PICP was effective on treating spontaneous chylothorax. Moreover, except for short-term of low fever and mild chest pain, no other adverse events had been found after PICP. Chest pain could be relieved by appeasement, feeding, and other ways to divert the children's attention, and fever could be relieved by physical and drug treatment. Of the cases involved, 14 children had anhelation and slightly decreased blood oxygen saturation, which was an effect of the compression of the affected lung and chest pain caused by the sclerosant; this was gradually alleviated and returned to normal after the sclerosant was drained out by the chest tube. Six children diagnosed with pneumonia were cured with antibiotic therapy. No cardiac, pulmonary, or renal dysfunction, dysthyroidism, visual impairment and recurrence of chylothorax were reported in the 22 patients who were followed up. Therefore, it can be considered that PICP is safe, minimally invasive and effective when the dosage of PI is properly controlled.

Our study has limitations. The result is not convincing enough based on a small sample size. Besides, we were not fully aware of the condition of the patient's first onset because most patients were transferred to our department from other hospitals. Furthermore, patients of different ages had different tolerances to CP treatment, and this may also happened to patients whom underwent multiple treatments, which resulted in different volumes of sclerosant used in our study. Therefore, the relationship between sclerosant dose and efficacy were not addressed in this study.

This study explored the improvement of PICP in the treatment of young pediatric patients with spontaneous chylothorax. Previous clinical experience can prove useful in the treatment of chylothorax. Chylothorax is a difficult and rare disease, which can impact the cardiopulmonary function, nutrition, and immunity of children. In recent years, with the continuous rich experience in the diagnosis and treatment of chylothorax, its mortality has gradually reduced to $10-20 \%$ (24). Some scholars have put forward the concept of multidisciplinary treatment of chylothorax $(25,26)$. Undoubtedly, the treatment of chylothorax needs the joint participation of pediatric, nutrition, cardiothoracic surgery, and related internal medical professionals. For the efficacy and safety of PI in the treatment of young children with spontaneous chylothorax, large-sample clinical research and further basic research are needed.

\section{Acknowledgments}

Funding: None.

\section{Footnote}

Reporting Checklist: The authors have completed the STROBE reporting checklist. Available at http://dx.doi. org/10.21037/apm-20-926

Data Sharing Statement: Available at http://dx.doi. org/10.21037/apm-20-926

Conflicts of Interest: All authors have completed the ICMJE uniform disclosure form (available at http://dx.doi. org/10.21037/apm-20-926). The authors have no conflicts of interest to declare.

Ethical Statement: The authors are accountable for all aspects of the work in ensuring that questions related to the accuracy or integrity of any part of the work are appropriately investigated and resolved. The research was in compliance of the Declaration of Helsinki and was approved by the ethical committee of Guangdong Second Provincial General Hospital (No. 20200407-01-YXKXYJ-LW). All of the enrolled patients' legal guardians were required to sign written informed consent prior to the treatment.

Open Access Statement: This is an Open Access article distributed in accordance with the Creative Commons Attribution-NonCommercial-NoDerivs 4.0 International License (CC BY-NC-ND 4.0), which permits the noncommercial replication and distribution of the article with the strict proviso that no changes or edits are made and the original work is properly cited (including links to both the 
formal publication through the relevant DOI and the license). See: https://creativecommons.org/licenses/by-nc-nd/4.0/.

\section{References}

1. Christofe NM, Pessotti CFX, Paiva L, et al. Incidence and Treatment of Chylothorax in Children Undergoing Corrective Surgery for Congenital Heart Diseases. Braz J Cardiovasc Surg 2017;32:390-3.

2. Papoulidis P, Vidanapathirana P, Dunning J. Chylothorax, new insights in treatment. J Thorac Dis 2018;10:S3976-7.

3. Lai Y, Zheng X, Yuan Y, et al. A modified pleurodesis in treating postoperative chylothorax. Ann Transl Med 2019;7:549.

4. Yoshida N, Baba H. Risk factors for chylothorax after esophagectomy. J Thorac Dis 2019;11:S196-7.

5. Tutor JD. Chylothorax in infants and children. Pediatrics 2014;133:722-33.

6. Le Tuan L, Nguyen Ngoc C, Tran Viet H, et al. An uncommon therapeutic option for a challenging cause of pleural effusion. Breathe (Sheff) 2019;15:e69-e76.

7. Zaki SA, Krishnamurthy MB, Malhotra A. Octreotide Use in Neonates: A Case Series. Drugs R D 2018;18:191-8.

8. Mierzejewski M, Korczynski P, Krenke R, et al. Chemical pleurodesis - a review of mechanisms in volved in pleural space obliteration. Respir Res 2019;20:247-53.

9. Chen $\mathrm{Y}, \mathrm{Li} \mathrm{C}, \mathrm{Xu} \mathrm{L}$, et al. Novel treatment for chylothorax after esophagectomy with $50 \%$ glucose pleurodesis. Ann Vasc Surg 2010;24:694.e9-13.

10. Cho HJ, Na KJ, Kim DW, et al. Chemical pleurodesis using a Viscum album extract in infants with congenital chylothorax. Eur J Pediatr 2014;173:823-6.

11. Brissaud O, Desfrere L, Mohsen R, et al. Congenital idiopathic chylothorax in neonates: chemical pleurodesis with povidone-iodine (Betadine). Arch Dis Child Fetal Neonatal Ed 2003;88:F531-3.

12. Lee CJ, Tsao PN, Chen CY, et al. Prenatal Therapy Improves the Survival of Premature Infants with Congenital Chylothorax. Pediatr Neonatol 2016;57:127-32.

13. Bender B, Murthy V, Chamberlain RS. The changing management of chylothorax in the modern era. Eur J Cardiothorac Surg 2016;49:18-24.

14. Yamakawa M, Doh SJ, Santosa SM, et al. Potential lymphangiogenesis therapies: Learning from current antiangiogenesis therapies - A review. Med Res Rev 2018;38:1769-98.
15. Sáinz-Jaspeado M, Claesson-Welsh L. Cytokines regulating lymphangiogenesis. Curr Opin Immunol 2018;53:58-63.

16. Agarwal R, Khan A, Aggarwal AN, et al. Efficacy \& safety of iodopovidone pleurodesis: a systematic review \& metaanalysis. Indian J Med Res 2012;135:297-304.

17. Matus I, Ho P. Ambulatory Iodopovidone Instillation Via Indwelling Pleural Catheters For Malignant Pleural Effusions. J Bronchology Interv Pulmonol 2019;26:290-2.

18. Bagheri R, Noori M, Rajayi M, et al. The effect of iodopovidone versus bleomycin in chemical pleurodesis. Asian Cardiovasc Thorac Ann 2018;26:382-6.

19. Borcyk K, Kamil A, Hagerty K, et al. Successful management of extremely high- output refractory congenital chylothorax with chemical pleurodesis using $4 \%$ povidone-iodine and propranolol: a case report. Clin Case Rep 2018;6:702-8.

20. Hmami F, Oulmaati A, Bouchikhi C, et al. Congenital chylothorax: rapid and complete response to polyvidone iodine. Arch Pediatr 2014;21:1002-5.

21. Murki S, Faheemuddin M, Gaddam P. Congenital chylothorax--successful management with chemical pleurodesis. Indian J Pediatr 2010;77:332-4.

22. Resch B, Freidl T, Reiterer F. Povidone-iodine pleurodesis for congenital chylothorax of the newborn. Arch Dis Child Fetal Neonatal Ed 2016;101:F87-8.

23. Lai Y, Zheng X, Yuan Y, et al. A modified pleurodesis in treating postoperative chylothorax. Ann Transl Med 2019;7:549.

24. Lin CH, Lin WC, Chang JS. Presentations and management of different causes of chylothorax in children: one medical center's experience. Biomedicine (Taipei) 2017;7:5.

25. Wiesner S, Uller W, Hofmann HS, et al. Complicated chylous pericardial and thoracic effusion as the first clinical manifestation of thoracic lymphatic malformation. Interact Cardiovasc Thorac Surg 2020;30:654-5.

26. Ball PL, Nethercott $S$, Beardsall K. Rare case of congenital chylothorax and challenges in its management. BMJ Case Rep 2019;12:e228023.

Cite this article as: Long WG, Cai B, Liu Y, Wang WJ. Povidone-iodine chemical pleurodesis in treating spontaneous chylothorax in pediatric patients. Ann Palliat Med 2020;9(3):10041012. doi: $10.21037 /$ apm-20-926 